\title{
Eduard GANS, Storia della Rivoluzione francese
}

Texte allemand et traduction italienne présentés par Corrado BERTANI, Milan, Rubbettino, 2011, ISBN 978-88-498-2844-3, $20 €$.

\section{Bernard Gainot}

\section{(2) OpenEdition Journals}

\section{Édition électronique}

URL : https://journals.openedition.org/ahrf/12906

DOI : 10.4000/ahrf.12906

ISSN : 1952-403X

\section{Éditeur :}

Armand Colin, Société des études robespierristes

\section{Édition imprimée}

Date de publication : 1 septembre 2013

Pagination : 222-223

ISBN : 9782200928261

ISSN : 0003-4436

Référence électronique

Bernard Gainot, «Eduard GAns, Storia della Rivoluzione francese », Annales historiques de la Révolution française [En ligne], 373 | juillet-septembre 2013, mis en ligne le 03 octobre 2013, consulté le 01 juillet 2021. URL : http://journals.openedition.org/ahrf/12906; DOI : https://doi.org/10.4000/ahrf.12906

Ce document a été généré automatiquement le 1 juillet 2021.

Tous droits réservés 


\section{Eduard GANS, Storia della Rivoluzione francese}

Texte allemand et traduction italienne présentés par Corrado BERTANI, Milan, Rubbettino, 2011, ISBN 978-88-498-2844-3, $20 €$.

\section{Bernard Gainot}

\section{RÉFÉRENCE}

Eduard GANS, Storia della Rivoluzione francese. Texte allemand et traduction italienne présentés par Corrado BERTANI, Milan, Rubbettino, 2011, ISBN 978-88-498-2844-3, $20 €$.

1 Cette édition est celle du cours d'Histoire délivré à l'Université de Berlin, pendant la saison d'été 1828 , à partir des notes prises par le futur compositeur Félix Mendelssohn Bartholdy, qui avait alors 19 ans.

2 Eduard Gans (1797 - 1839) est né à Berlin dans une famille juive aisée. Il fait des études de droit, notamment à Heidelberg, où il suit les cours de Hegel, dont il devient l'un des disciples les plus marquants. Il se convertit en 1825 à l'Église évangélique de Prusse. La même année, il fait un séjour à Paris, où il fréquente les cercles et salons sous la conduite de Victor Cousin. Lorsqu'il prononce son cours de 1828, il est professeur à la Faculté de droit de l'Université de Berlin. Ce cours sur la Révolution française, découpé en six sections chronologiques, devait se prolonger par une «Histoire des cinquante dernières années ", dont les notes sont transcrites dans la dernière partie du présent ouvrage. La tonalité libérale devait fortement déplaire au gouvernement prussien, qui l'interdit peu avant sa mort.

3 Les lignes directrices du cours sont les caractères fondamentaux de l'institution monarchique, le problème de la souveraineté, les rapports entre les différents pouvoirs, les conséquences de l'irruption des masses sur la scène politique.

4 Gans applique à l'Histoire contemporaine les principes de la philosophie de Hegel, en réaction aux tendances moralisantes qui dominaient alors l'approche historique 
«pragmatique ». Il s'agit de mettre au jour la logique sous-jacente à l'exposé des faits : le processus par lequel l'esprit, en transformant le réel, prend progressivement conscience de lui-même et de sa liberté essentielle. Il reprend les propositions de Hegel sur le cours de l'Histoire universelle, à travers les étapes fondamentales que sont la Réforme luthérienne, la Révolution anglaise, et la Révolution française comme événement décisif pour l'entrée de l'Europe dans l'histoire contemporaine.

5 Sa documentation repose surtout sur l'utilisation de l'ouvrage de Mignet, de préférence à celui de Thiers, trop factuel à son goût. La méthode de Mignet, plus conceptuelle, convient davantage à son propos, même s'il prend des distances avec la causalité. Il est attentif à la logique sous-jacente des processus révolutionnaires, là où Mignet mettait plutôt en avant la causalité sociale. Pour ce dernier c'est la résistance forcenée des couches privilégiées qui induit la radicalité du cours de la Révolution et sa popularisation. Le caractère de " nécessité » de la Révolution se comprend chez Mignet comme l'obligation de prendre des mesures de salut public pour sauver les acquis des premières années; chez Gans, c'est un effet de la nature logique des premières mesures, qui conduisent inéluctablement, et presque indépendamment de la volonté des acteurs et des groupes, vers les mesures extrêmes.

En outre, contrairement à Mignet, Gans n'est pas lié par le contexte français de la Restauration. La contingence politique conduit Mignet à une écriture contre-factuelle ; libéral et partisan de la monarchie constitutionnelle, ce dernier s'attache à montrer que le sort de l'institution monarchique eut été différent si les Bourbons avaient d'emblée lié leur destin à la mise en œuvre des réformes, plutôt qu'à la résistance désespérée des privilégiés. Gans est surtout attaché à une démarche pédagogique pour un auditoire prussien et, ce faisant, il s'attache à l'examen des grandes catégories de référence pour la construction de l'État moderne en Europe.

7 Ces catégories sont d'abord la souveraineté, puis la représentation, enfin la liberté politique. A partir de là, il interprète la période révolutionnaire (son exposé se termine à l'avènement du Directoire) comme une crise de légitimité. Les prémices de la dérive jacobine sont tout entiers contenus dans la stricte séparation des pouvoirs opérée par les Constituants. Attaché à une notion absolue de la souveraineté qui doit plus à Bodin qu'à Locke, Gans, tout comme son maître Hegel, relève la contradiction entre la rationalité à l'œuvre dans la permanence de l'indivisibilité de l'État, et le principe de représentation de la Nation tel qu'il fut mis en œuvre par les constituants. Certes, Gans sait gré à ceux-ci d'avoir prohibé le mandat impératif pour exprimer une "volonté générale » indépendante des intérêts subjectifs. Toutefois, la volonté objective qui se déploie lors de la délibération en commun doit composer avec la volonté subjective qui se déploie lors des élections en assemblées primaires. La politique des intérêts ressurgit avec cette interprétation numérique de la majorité et, avec elle, la division des partis. $\mathrm{Et}$, de ce point de vue, Gans condamne tout aussi bien les restrictions censitaires du suffrage, qui redoublent les inconvénients de la majorité numérique par une subordination aux intérêts matériels. Pour lui, l'Homme ne peut pleinement exercer sa liberté politique que si le gouvernement reste un point de référence fixe (ce qui suppose une monarchie héréditaire), composé de fonctionnaires inamovibles choisis par le monarque. C'est un gouvernement de techniciens assisté d'un corps délibératif composé selon la répartition de la société en «états ». On mesure toute la distance entre ce constitutionnalisme rationnel, et le constitutionnalisme libéral classique. 
8 Gans va toutefois évoluer sur la question après 1830. Il cherche à réintroduire une dose de suffrage sur une base numérique, qui est celle de la proportionnalité de la représentation au nombre des habitants. Il se rallie ensuite au principe de la prise de décision à la majorité, puis à l'idée d'une opposition parlementaire. Gans cherche une voie de compromis entre les vues de Hegel et le système représentatif, plus à même de répercuter les transformations sociales à l'ouvre dans les États modernes. Il ne se réfère plus au «bourgeois » lié à son activité professionnelle et à une communauté restreinte, mais au «citoyen » bien informé des réalités nationales, mieux à même de réaliser la médiation entre la sphère de la société civile et l'universalité de la loi. Évolution qui lui valut les tracasseries de la fin de sa vie?

9 Le cours de 1828, à travers les notes de Mendelssohn, est donc reproduit dans une édition bilingue, allemande sur la page de gauche, italienne sur la page de droite, avec un appareil érudit tout à fait considérable ; soixante-dix pages de notes qui éclairent le sens des notions utilisées et des événements, et qui replacent les enjeux interprétatifs à la lumière de l'historiographie récente de la Révolution française.

10 Le cours est complété par les quatre leçons sur l'histoire des cinquante dernières années, qui replacent la signification de la Révolution française dans l'histoire européenne, qui exprimait alors toute l'histoire universelle.

11 C'est une très belle et très heureuse initiative, référence essentielle pour tous ceux qui s'intéressent à l'historiographie de la Révolution française et, plus précisément aux traces multiples que celle-ci a laissées pour le mouvement intellectuel du Vormärz allemand. 